\title{
Computing Cusps of 3R Robots Using Distance Geometry
}

\author{
Federico Thomas
}

\begin{abstract}
The singularities of a 3R robot are usually determined, in terms of its joint angles, from the determinant of its Jacobian which can then be mapped onto the robot's workspace through its forward kinematics. The presence of cusps in these singularity plots permits to change robot's posture without meeting a singularity and hence their relevance. This paper shows how, using Distance Geometry, the singularities in the workspace of a $3 \mathrm{R}$ robot can be represented as an octic curve of the form $4 \delta_{1} \delta_{3}-\delta_{2}^{2}=0$, where $\delta_{i}, i=1,2,3$, are quartic polynomials and, what is more important, its cusps correspond to those points in which $\delta_{2}=\delta_{3}=0$. This leads to important simplifications over previous approaches.
\end{abstract}

Key words: $3 \mathrm{R}$ regional robot, wrist-partitioned robots, cuspidal robots, Distance Geometry.

\section{Introduction}

The presence of cusps in the singularity locus of a 3R robot allows it to change posture without meeting a singularity (see [1] and the references therein). Cusps correspond to points of the workspace where the robot's inverse kinematics admit three equal solutions, as it was first established in [2]. Then, the existence of cusps is usually determined from its fourth-degree inverse kinematics polynomial, though other alternatives are possible [3]. The symbolic condition, in terms of the $\mathrm{DH}$ parameters of the robot, for this polynomial to have three equal roots has been considered as intractable [1]. Only the case of orthogonal 3R robots - robots whose consecutive joint axes are mutually orthogonal — has been analyzed in detail [4]. This paper puts forward a new formulation of the problem that could lead to new insights into

Federico Thomas

Institut de Robòtica i Informàtica Industrial (CSIC-UPC) e-mail: fthomas@ iri.upc.edu 
the general case thanks to the simplicity and symmetry of the resulting algebraic expressions.

This paper is organized as follows. Section 2 describes how, using Distance Geometry, the inverse kinematics of a $3 \mathrm{R}$ robot boils down to compute the intersection of two ellipses. Section 3 and Section 4 show how the singularity locus of a 3R robot can be computed from the pencil of conics defined by these two ellipses, and how cusps in this locus correspond to osculating contacts between them. An example is presented in Section 5, and some conclusions are drawn in Section 6.

\section{Distance-based formulation}

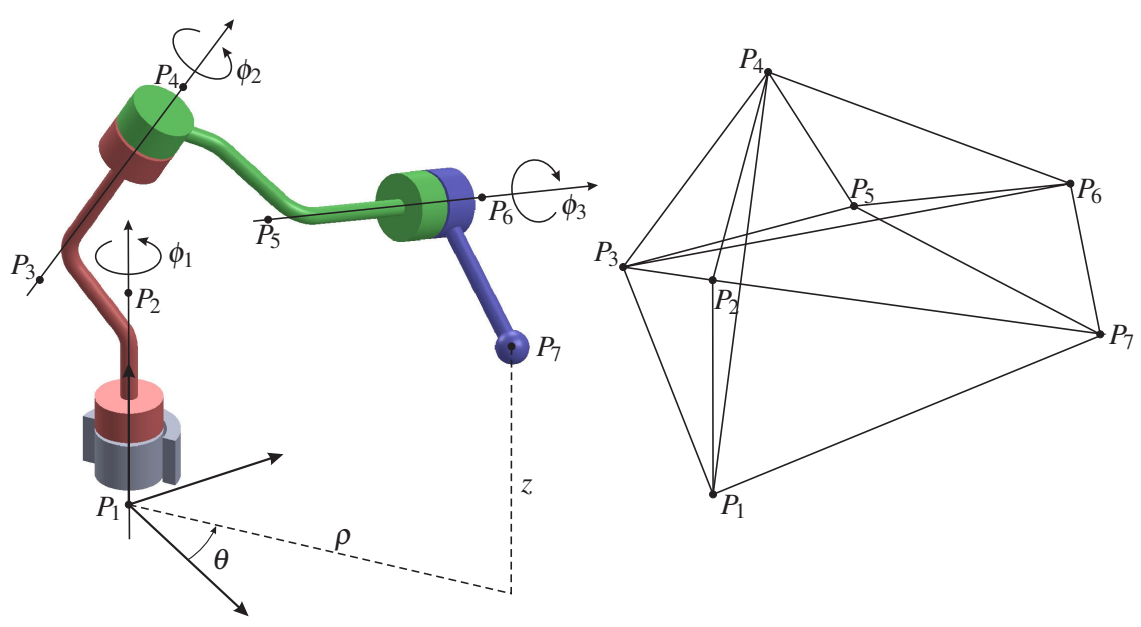

Fig. 1 A 3R regional robot (left) and its associated bar-and-joint framework (right).

A regional 3R robot is an open chain of four rigid bodies (the links), pairwise articulated through revolute joints [Fig. 1(left)]. The inverse kinematics problem is to find all valid configurations of this chain $\left(\phi_{1}, \phi_{2}\right.$, and $\left.\phi_{3}\right)$ that are compatible with a specified location for $P_{7}$, relative to a global reference frame. Then, the first and last links can be seen as articulated through a spherical joint centered at $P_{7}$ and the problem is equivalent to that of finding the valid configurations of a closed loop of four pairwise articulated links through three revolute joints and one spherical joint.

A link connecting two revolute axes can be modeled by taking two points on each of these axes, and by connecting them all with rigid bars to form a tetrahedron. A link connecting a revolute axis and a spherical joint can be modeled by taking two points on the revolute joint axis and the center of the spherical joint, and by connecting them all with rigid bars to form a triangle. In this way, a $3 \mathrm{R}$ regional 
robot can be modeled as the bar-and-joint framework shown in Fig. 1(right). In this conversion we are loosing an important information: the orientation of the two tetrahedra. Nevertheless, given a valid configuration of the obtained bar-and-joint framework, we can derive another valid configuration with the desired orientations for the two tetrahedra. Indeed, observe that $P_{3}, P_{4}$, and $P_{7}$ defines a plane that divides the framework in two halves and the mirror projection of any of these two halves with respect to this plane changes the orientation of the corresponding tetrahedron while preserving the bar lengths. As a consequence, any valid configuration for the framework translates into a valid configuration for the $3 \mathrm{R}$ robot.

The distances between the set of points $\left\{P_{1}, P_{2}, P_{3}, P_{4}, P_{7}\right\}$ or $\left\{P_{3}, P_{4}, P_{5}, P_{6}, P_{7}\right\}$ are not independent because they are embedded in $\mathbb{R}^{3}$. This dependency, using the theory of Cayley-Menger determinants, translates into the following algebraic conditions:

$$
\left|\begin{array}{cccccc}
0 & 1 & 1 & 1 & 1 & 1 \\
1 & 0 & s_{1,2} & s_{1,3} & s_{1,4} & s_{1,7} \\
1 & s_{2,1} & 0 & s_{2,3} & s_{2,4} & s_{2,7} \\
1 & s_{3,1} & s_{3,2} & 0 & s_{3,4} & s_{3,7} \\
1 & s_{4,1} & s_{4,2} & s_{4,3} & 0 & s_{4,7} \\
1 & s_{7,1} & s_{7,2} & s_{7,3} & s_{7,4} & 0
\end{array}\right|=0 \text { and }\left|\begin{array}{cccccc}
0 & 1 & 1 & 1 & 1 & 1 \\
1 & 0 & s_{3,4} & s_{3,5} & s_{3,6} & s_{3,7} \\
1 & s_{4,3} & 0 & s_{4,5} & s_{4,6} & s_{4,7} \\
1 & s_{5,3} & s_{5,4} & 0 & s_{5,6} & s_{5,7} \\
1 & s_{6,3} & s_{6,4} & s_{6,5} & 0 & s_{6,7} \\
1 & s_{7,3} & s_{7,4} & s_{7,5} & s_{7,6} & 0
\end{array}\right|=0,
$$

where $s_{i, j}$ stands for the squared distance between $P_{i}$ and $P_{j}$. The above two equations are quadratic forms in the unknown distances $s_{3,7}$ and $s_{4,7}$. They actually represent two real ellipses, $\mathscr{A}: \mathbf{x A \mathbf { A }} \mathbf{T}^{T}=0$ and $\mathscr{B}: \mathbf{x B x}^{T}=0$, where $\mathbf{x}=\left(s_{3,7}, s_{4,7}, 1\right)$ and

$$
\mathbf{A}=\left(\begin{array}{lll}
a_{1} & c_{1} & d_{1} \\
c_{1} & b_{1} & e_{1} \\
d_{1} & e_{1} & f_{1}
\end{array}\right) \text { and } \mathbf{B}=\left(\begin{array}{lll}
a_{2} & c_{2} & d_{2} \\
c_{2} & b_{2} & e_{2} \\
d_{2} & e_{2} & f_{2}
\end{array}\right)
$$

The entries $d_{1}$ and $e_{1}$ depend linearly on $s_{1,7}$ and $s_{2,7}$, while $f_{1}$ depends on them quadratically. All other entries of $\mathbf{A}$ and $\mathbf{B}$ are constant for a given robot and they can be expressed in terms of determinants of known bar lengths (see [5] for details).

Solving the inverse kinematics of a $3 \mathrm{R}$ robot consists in obtaining the sets of joint angles $\left(\phi_{1}, \phi_{2}, \phi_{3}\right)$ that provide the desired position of its end-effector given in cylindrical coordinates by $(\theta, \rho, z)$. Due to the symmetry with respect to the first joint axis, $\phi_{1}$ is trivially obtained. The other two cylindrical coordinates, $\rho$ and $z$, determine the squared distances $s_{1,7}$ and $s_{2,7}$. Then, we can compute the intersection of $\mathscr{A}$ and $\mathscr{B}$ to obtain up to four sets of distances $\left(s_{3,7}, s_{4,7}\right)$ compatible with all other distances. It is important to note that, given the orientation of the tetrahedra with vertex sets $\left\{P_{1}, P_{2}, P_{3}, P_{4}\right\}$ and $\left\{P_{3}, P_{4}, P_{5}, P_{6}\right\}$, every solution for $\left(s_{3,7}, s_{4,7}\right)$ leads to a unique solution for $\left(\phi_{2}, \phi_{3}\right)$. Thus, solving the inverse kinematics of a $3 \mathrm{R}$ regional robot reduces to calculate the intersections of two ellipses. 


\section{Singularities}

A singularity occurs when we have a repeated solution of the inverse kinematics, that is, when $\mathscr{A}$ and $\mathscr{B}$ are tangent. The positional relationship between $\mathscr{A}$ and $\mathscr{B}$ can be derived from the study of the pencil of conics they define, that is, from the family of conics defined by $\mathbf{p}^{T}(\lambda \mathbf{A}+\mathbf{B}) \mathbf{p}=0, \lambda \in \mathbb{R}$ (see [6] for an introductory explanation). The values of $\lambda$ for which a conic of this pencil is degenerate correspond to those in which

$$
f(\lambda)=\operatorname{det}(\lambda \mathbf{A}+\mathbf{B})=l_{3} \lambda^{3}+3 l_{2} \lambda^{2}+3 l_{1} \lambda+l_{0}=0,
$$

where the coefficients $l_{i}, i=0,1,2,3$, can expressed in a neat and elegant way as [7, p. 191]:

$$
\begin{aligned}
l_{3} & =\left|\begin{array}{lll}
a_{1} & c_{1} & d_{1} \\
c_{1} & b_{1} & e_{1} \\
d_{1} & e_{1} & f_{1}
\end{array}\right|=\operatorname{det}(\mathbf{A}), \\
3 l_{2} & =\left|\begin{array}{lll}
a_{2} & c_{1} & d_{1} \\
c_{2} & b_{1} & e_{1} \\
d_{2} & e_{1} & f_{1}
\end{array}\right|+\left|\begin{array}{lll}
a_{1} & c_{2} & d_{1} \\
c_{1} & b_{2} & e_{1} \\
d_{1} & e_{2} & f_{1}
\end{array}\right|+\left|\begin{array}{lll}
a_{1} & c_{1} & d_{2} \\
c_{1} & b_{1} & e_{2} \\
d_{1} & e_{1} & f_{2}
\end{array}\right|, \\
3 l_{1} & =\left|\begin{array}{lll}
a_{1} & c_{2} & d_{2} \\
c_{1} & b_{2} & e_{2} \\
d_{1} & e_{2} & f_{2}
\end{array}\right|+\left|\begin{array}{lll}
a_{2} & c_{1} & d_{2} \\
c_{2} & b_{1} & e_{2} \\
d_{2} & e_{1} & f_{2}
\end{array}\right|+\left|\begin{array}{lll}
a_{2} & c_{2} & d_{1} \\
c_{2} & b_{2} & e_{1} \\
d_{2} & e_{2} & f_{1}
\end{array}\right|, \\
l_{0} & =\left|\begin{array}{lll}
a_{2} & c_{2} & d_{2} \\
c_{2} & b_{2} & e_{2} \\
d_{2} & e_{2} & f_{2}
\end{array}\right|=\operatorname{det}(\mathbf{B}) .
\end{aligned}
$$

The above polynomial in $\lambda$ is known as the generalized characteristic polynomial of the pencil. By definition, $f(\lambda)=0$ has a multiple root if, and only if, its discriminant, say $\Delta$, vanishes. Furthermore, it can be shown that $f(\lambda)=0$ has three simple real roots if $\Delta>0$, and $f(\lambda)=0$ has two complex conjugate roots and a real root if $\Delta<0$. The roots of $\Delta=0$ give information on the positional relationship between $\mathscr{A}$ and $\mathscr{B}$. Actually, the sign of the discriminant $\Delta$ gives information about the order of accessibility of the robot's workspace. It permits to decompose it in the following three regions:

- $\Delta<0$ corresponds to a two-way accessible region;

- $\Delta=0$ corresponds to the singularities of the robot; and

- $\Delta>0$ corresponds to a four-way accessible region or an inaccessible region.

The standard expression of the discriminant of a cubic gives little insight into the structure of our problem. Fortunately, a much more convenient expression, that will reveal very important for the computation of cusps, can be found in [8]. It reads as follows:

$$
\Delta=\left|\begin{array}{cc}
2 \delta_{1} & \delta_{2} \\
\delta_{2} & 2 \delta_{3}
\end{array}\right|
$$


where

$$
\delta_{1}=\left|\begin{array}{ll}
l_{3} & l_{2} \\
l_{2} & l_{1}
\end{array}\right|, \delta_{2}=\left|\begin{array}{ll}
l_{3} & l_{1} \\
l_{2} & l_{0}
\end{array}\right|, \delta_{3}=\left|\begin{array}{ll}
l_{2} & l_{1} \\
l_{1} & l_{0}
\end{array}\right|
$$

Observe that the condition of singularity $\Delta=0$ is expressed as a determinant of determinants of determinants of determinants (four levels of nested determinants). It can be shown that the elements of the third level of determinants depend quadratically on $s_{3,7}$ and $s_{4,7}$. Then, since the two outer levels of determinants are quadratic with respect to their elements, the singularity locus can be displayed as a curve of order $2^{3}$ in the plane defined by $s_{3,7}$ and $s_{4,7}$.

\section{Cusps as osculating contacts}

If three of the points of intersection between $\mathscr{A}$ and $\mathscr{B}$ coincide, the ellipses are said to osculate each other at this point. In this case, the characteristic polynomial of the pencil they define is a perfect cube [9]. As a consequence,

$$
\frac{l_{3}}{l_{2}}=\frac{l_{2}}{l_{1}}=\frac{l_{1}}{l_{0}}=-\frac{1}{r}
$$

where $r$ is the triple root of the characteristic polynomial. Then, observe that

$$
\frac{l_{3}}{l_{2}}=\frac{l_{2}}{l_{1}} \Rightarrow \delta_{1}=0, \frac{l_{3}}{l_{2}}=\frac{l_{1}}{l_{0}} \Rightarrow \delta_{2}=0, \text { and } \frac{l_{2}}{l_{1}}=\frac{l_{1}}{l_{0}} \Rightarrow \delta_{3}=0 .
$$

In other words, in a cusp not only $\Delta=0$, but all three components of the discriminant vanish. Moreover,

$$
\begin{aligned}
& \left.\begin{array}{l}
\delta_{1}=0 \\
\delta_{2}=0
\end{array}\right\} \Rightarrow \Delta=0 \text {, and } \delta_{3}=0 \text { iff } \frac{l_{2}}{l_{3}} \neq \frac{0}{0}, \\
& \left.\begin{array}{l}
\delta_{2}=0 \\
\delta_{3}=0
\end{array}\right\} \Rightarrow \Delta=0 \text {, and } \delta_{1}=0 \text { iff } \frac{l_{0}}{l_{1}} \neq \frac{0}{0} \text {. }
\end{aligned}
$$

Since $l_{0}=\operatorname{det}(\mathbf{B}) \neq 0$ by construction, we conclude that we have a cusp if, and only if, $\delta_{2}=0$ and $\delta_{3}=0$.

\section{Example}

Let us considered the orthogonal 3R robot with the standard DH parameters $d_{1}=1$, $d_{2}=1 / 2, d_{3}=1, a_{1}=1, a_{2}=4 / 5$ and $a_{3}=2$. Then, by locating $P_{1}$ and $P_{7}$ at the origin of the reference frame and at the center of the end-effector, respectively; $P_{2}$ and $P_{3}$ on the common normal between the first and the second axis; $P_{4}$ and $P_{5}$ on the common normal between the second and the third revolute axis; and $P_{6}$ as the nearest 


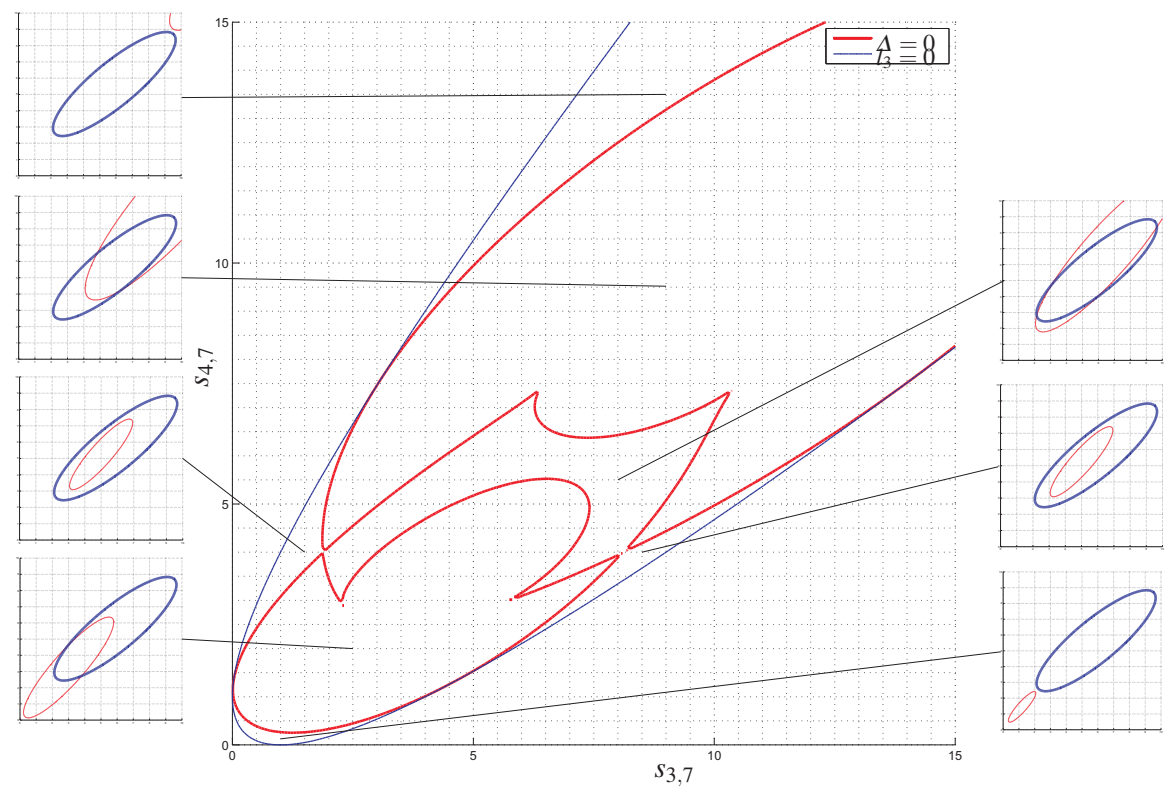

Fig. 2 The singularity locus $(\Delta=0)$ divides the area where $l_{3}<0$ (the region where $\mathscr{A}$ is a real ellipse) into regions where the spatial relationship between $\mathscr{A}$ and $\mathscr{B}$ is the same.

point to $P_{7}$ on the third revolute axis, we have that $s_{1,2}=d_{1}^{2}, s_{2,3}=a_{1}^{2}, s_{3,4}=d_{2}^{2}$, $s_{4,5}=a_{2}^{2}, s_{5,6}=d_{3}^{2}, s_{6,7}=a_{3}^{2}$. Then, by Pythagoras' theorem, $s_{1,3}=s_{1,2}+s_{2,3}$, $s_{2,4}=s_{2,3}+s_{3,4}, s_{3,5}=s_{3,4}+s_{4,5}, s_{4,6}=s_{4,5}+s_{5,6}, s_{5,7}=s_{5,6}+s_{6,7}$. Moreover, since $\alpha_{2}=\pi / 2$ and $\alpha_{3}=\pi / 2, s_{1,4}=s_{1,3}+s_{3,4}, s_{3,6}=s_{3,5}+s_{5,6}$.

Substituting the above squared distances in the expressions for $\mathscr{A}$ and $\mathscr{B}$, we obtain:

$$
\left(\begin{array}{lll}
s_{3,7} & s_{4,7} & 1
\end{array}\right)\left(\begin{array}{ccc}
5 & -4 & d \\
-4 & 4 & e \\
d & e & f
\end{array}\right)\left(\begin{array}{c}
s_{3,7} \\
s_{4,7} \\
1
\end{array}\right)=0
$$

and

$$
\left(\begin{array}{lll}
s_{3,7} & s_{4,7} & 1
\end{array}\right)\left(\begin{array}{ccc}
2.56 & -2.56 & -0.64 \\
-2.56 & 3.56 & -5 \\
-0.64 & -5 & 21.7296
\end{array}\right)\left(\begin{array}{c}
s_{3,7} \\
s_{4,7} \\
1
\end{array}\right)=0
$$

respectively, where $d, e$, and $f$ depend on $s_{1,7}$ and $s_{2,7}$ as follows:

$$
\left(\begin{array}{l}
d \\
e
\end{array}\right)=\left(\begin{array}{ccc}
0 & -1 & 0 \\
0 & 0 & -1
\end{array}\right)\left(\begin{array}{c}
s_{1,7} \\
s_{2,7} \\
1
\end{array}\right) \text { and } f=\left(\begin{array}{lll}
s_{1,7} & s_{2,7} & 1
\end{array}\right)\left(\begin{array}{ccc}
1 & -1 & -1 \\
-1 & 2 & 0 \\
-1 & 0 & 2.25
\end{array}\right)\left(\begin{array}{c}
s_{1,7} \\
s_{2,7} \\
1
\end{array}\right) .
$$




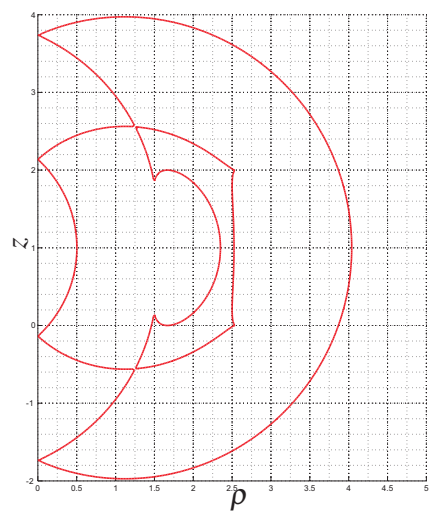

Fig. 3 The singularity locus represented in Fig. 2 mapped onto the robot's workspace. Both plots are in one-to-one correspondence.

Now, substituting in (4)-(7), we obtain:

$$
\begin{aligned}
l_{0} & =-\frac{16384}{625} \\
l_{1} & =\frac{18848}{1875} d+\frac{64}{75} f+\frac{263116}{46875} \\
& =\frac{64}{75} s_{1,7}^{2}-\frac{128}{75} s_{1,7} s_{2,7}-\frac{128}{75} s_{1,7}+\frac{128}{75} s_{2,7}^{2}-\frac{18848}{1875} s_{2,7}-\frac{173116}{46875} \\
l_{2} & =\frac{1256}{75} d+\frac{63}{25} f-\frac{89}{75} d^{2}+\frac{18274}{1875} \\
& =\frac{63}{25} s_{1,7}^{2}-\frac{126}{25} s_{1,7} s_{2,7}-\frac{126}{25} s_{1,7}+\frac{289}{75} s_{2,7}^{2}-\frac{1256}{75} s_{2,7}+\frac{115621}{7500} \\
l_{3} & =-4 d^{2}+8 d+4 f-5 \\
& =4 s_{1,7}^{2}-8 s_{1,7} s_{2,7}-8 s_{1,7}+4 s_{2,7}^{2}-8 s_{2,7}+4
\end{aligned}
$$

Then, substituting in (9), we finally obtain:

$$
\begin{aligned}
\delta_{1}= & \frac{4814}{1875} d^{2} f-\frac{1184996}{15625} f-\frac{70064}{1875} d f-\frac{59287072}{140625} d-\frac{7239812}{46875} d^{2} \\
& -\frac{2608}{5625} d^{3}-\frac{7921}{5625} d^{4}-\frac{5507}{1875} f^{2}-\frac{235270576}{3515625} \\
\delta_{2}= & \frac{5696}{5625} d^{2} f-\frac{348149476}{3515625} f-\frac{5571872}{140625} d f-\frac{250411552}{1171875} d-\frac{82201508}{1171875} d^{2} \\
& +\frac{1677472}{140625} d^{3}-\frac{1344}{625} f^{2}+\frac{16328181784}{87890625} \\
\delta_{3}= & -\frac{28665899264}{87890625} d-\frac{198564352}{3515625} f-\frac{2412544}{140625} d f-\frac{245883904}{3515625} d^{2} \\
& -\frac{4096}{5625} f^{2}-\frac{630607309456}{2197265625}
\end{aligned}
$$


The robot's singularity locus is given by $\Delta=4 \delta_{1} \delta_{3}-\delta_{2}^{2}=0$. This locus segments the plane define by $\left(s_{3,7}, s_{4,7}\right)$ into regions with the same number of inverse kinematics solutions (Fig. 2). It can be mapped onto the robot's workspace by observing that $z=\left(d_{1,2}^{2}-s_{2,7}+s_{1,7}\right) / 2 d_{1,2}$ and $\rho=+\sqrt{s_{2,7}-\left(d_{1,2}-z\right)^{2}}$, where $d_{1,2}=\sqrt{s_{1,2}}=1$. The result is represented in Fig. 3.

The curves defined by $\delta_{2}=0$ and $\delta_{3}=0$ are plotted in Fig. 4. Observe how their intersection coincide with the cusps of the singularity locus plotted in Fig. 2. The system of equations $\delta_{2}=\delta_{3}=0$ has 12 solutions, 8 of them are complex. The real solutions correspond to the coordinates of the 4 cusps: $(5.4364,2.8669)$, (10.4444, 7.4444), (2.2975, 2.8669), and (6.4444, 7.4444).

We have analyzed an orthogonal $3 \mathrm{R}$ robot and this induces some simplifications in the derived equations. For example, $\delta_{3}$ is cubic with respect to $s_{1,7}$ and $s_{2,7}$ instead of quartic (as is the case in general). This leads to 12 solutions for the system $\delta_{2}=$ $\delta_{3}=0$ while, in general, we would have 16 .

\section{Conclusion}

We have shown how the kinematics of a $3 \mathrm{R}$ robot is determined by the positional relationship between two ellipses, and how cusps in the singularity locus correspond to osculating contacts between these two ellipses. This geometric interpretation translates into an algebraic characterization of cusps as the intersection of two quartic curves, a simpler characterization than the traditional one based on imposing three equal roots to the inverse kinematics polynomial.

\section{References}

1. Wenger, P.: Cuspidal and noncuspidal robot manipulators. Robotica 25(6), 677-689 (2007)

2. El Omri, J., and Wenger, P.: How to recognize simply a non-singular posture changing 3-DOF manipulator. Proc. 7th ICAR, 215-222 (1995)

3. Husty, M., Ottaviano, E., and Ceccarelli, M.: A geometrical characterization of workspace singularities in 3R manipulators. In Advances in Robot Kinematics: Analysis and Design, J. Lenarčič and P. Wenger (Eds.), Springer, 411-418 (2008)

4. Baili, M., Wenger, P., and Chablat, D.: A classification of $3 R$ orthogonal manipulators by the topology of their workspace. IEEE Int. Conf. on Robotics and Automation (2004)

5. Thomas, F., Re-examining the singularity loci of 3R manipulators. In preparation.

6. Choi, Y-K., Wang, W., Liu, Y., and Kim, M-S.: Continuous collision detection for two moving elliptic disks. IEEE Trans. on Robotics 22(2), 213-224 (2006)

7. Richter-Gebert, J.: Perspectives on Projective Geometry: A Guided Tour Through Real and Complex Geometry. Springer (2011)

8. Blinn, J.F.: Polynomial discriminants I. Matrix magic. IEEE Computer Graphics and Applications 20(6), 94-98 (2002)

9. Salmon, G.: A Treatise on Conic Sections, Chelsea Publishing Co. (1869) 